\title{
Ceux qui sont proches : les soigneurs au zoo
}

Jean Estebanez ${ }^{1}$

Résumé : Cet article propose d'analyser le rôle des soigneurs au zoo et la façon dont leur position éclaire les relations entre humains et animaux. Construit autour de terrains dans trois zoos français, il analyse le rôle de la distance dans la définition de ce qu'est être soigneur. La proximité semble leur conférer - dans leur discours ou dans le regard que portent sur eux le public - une connaissance vraie des animaux; elle les expose également à un risque de blessure important. La distance enfin joue sur la définition même des acteurs en présence. Animaux et soigneurs se redéfinissent mutuellement par leur proximité.

Mots-clés : Soigneur, Humain, Animaux, Distance, Catégories

\begin{abstract}
This paper analyses zookeepers in three french zoos and the prospects their work gives us on human-animal relationship. Distance is central to their definition: they are the closest one to animals and therefore the most knowledgeable about them to their mind but also to the public one. Distance has an impact on keepers and animals nature. Are animals close to humans still wild? How does it come that people throw pop corn to keepers when they are in a cage?
\end{abstract}

Keywords: Zookeepers, Human, Animals, Distance, Categories

\footnotetext{
1 Doctorant en géographie, Université de Paris Diderot/Université de Genève, Jean.Estebanez@ens.fr
} 
Les zoos sont des institutions de taille très variable, d'une ou deux personnes dans les plus petites structures, à plusieurs centaines de salariés dans des institutions comme le zoo de San Diego, aux Etats-Unis. Dans les plus petits d'entre eux, la pluriactivité du travail est la norme. Il y a toujours cependant quelques tâches comme nourrir les animaux ou nettoyer les enclos qui doivent êtres accomplies au quotidien, tout au long de l'année. Ceux qui s'en occupent sont ainsi un rouage essentiel des zoos.

Leur rôle nous aide-t-il pour autant à comprendre quelque chose à ce qui s'y joue ? Ne sont-ils pas comme les machinistes à l'opéra, essentiels pour le mouvement des décors, mais dont il est difficile de dire qu'ils font le sens du spectacle?

Si on doit réduire un zoo à sa plus simple expression, n'est-ce pas plutôt un lieu où se retrouvent des animaux et un public ? En somme qu'a-t-on à apprendre des soigneurs ?

L'hypothèse qui fonde cet article est qu'un des enjeux essentiels au zoo est le rapport à la distance. Entre humains et animaux, le zoo organise et négocie une juste distance. J'essayerai ici de montrer que les soigneurs ont une position particulière dans ce jeu, qui nous éclaire, audelà de leur métier, sur la fonction du zoo. Si relativement peu de travaux sont consacrés aux zoos en sciences humaines (voir, par exemple, Anderson, 1995 ; Batary É., Hadouin-Fugier É., 1998 ; Berger, 1980 ; Estebanez, 2006 ; Mullan, Marvin, 1998 ; Rothfeld, 2002), ceux qui s'intéressent aux soigneurs sont quasiment inexistants (voir cependant Pellegrini, 1995, p. 234-235).

Cette étude se fonde sur trois terrains dans des zoos français : Zoodyssée, près de Niort (lundi 16-mardi 22 juillet 2007) ; zoo de Pont-Scorff (lundi 26 juillet-mercredi 1 août 2007) et la Ménagerie du Jardin des Plantes à Paris (lundi 3 septembre-vendredi 14 septembre 2007). Le texte renvoie donc avant tout au cas français. On trouverait des différences notables en s'intéressant par exemple aux soigneurs aux Etats-Unis, dont la position dans la hiérarchie des zoos est très différente. A l'occasion de mes terrains j'ai suivi de l'intérieur la vie des différents services des trois zoos et en particulier les soigneurs. Chaque demi-journée m'a permis de suivre une équipe différente et de connaître au final l'ensemble du personnel de l'institution que je visitais. J'ai en particulier documenté les pratiques des acteurs par des photographies et des notes, tout en leur posant des questions générales sur le sens du métier, ce qui leur plaît et leur déplaît, leur formation... J'ai ainsi constitué un corpus de 850 photographies et un journal de 589 pages, pour moitié composé de notes de terrain et pour moitié composé d'un premier décryptage, effectué le jour même ou au plus tard dans la semaine.

\section{Soigneurs et animaux : la fonction de la distance}




\section{La distance et le sens de la profession}

Etre soigneur est un métier éprouvant physiquement, aux horaires lourds, surtout dans les parcs privés où les semaines peuvent atteindre 70 heures et payé au SMIC. A quelques

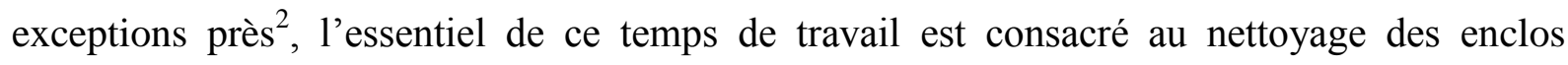
(ramassage des excréments et balayage). Pourtant, le métier attire et beaucoup de jeunes soigneurs passent de nombreuses années en contrat à durée indéterminée et/ou à temps partiel. On ne devient pas soigneur par hasard, même si un certain nombre d'entre eux parlent d'études de vétérinaires qu'ils n'ont pas réussi à suivre. Devenir soigneur, c'est s'assurer une place auprès d'animaux non domestiques et souvent exotiques ${ }^{3}:$ « Nettoyer le parc des émeus vide, c'est du balayage. Quand ils y sont, c'est autre chose [...] On a pas l'impression d'être au milieu de la savane australienne, mais presque [...]. Au début [de mon travail], j'étais un peu fou : on peut rentrer avec qui, avec quoi ?» ${ }^{4}$ (cf. figure 1).

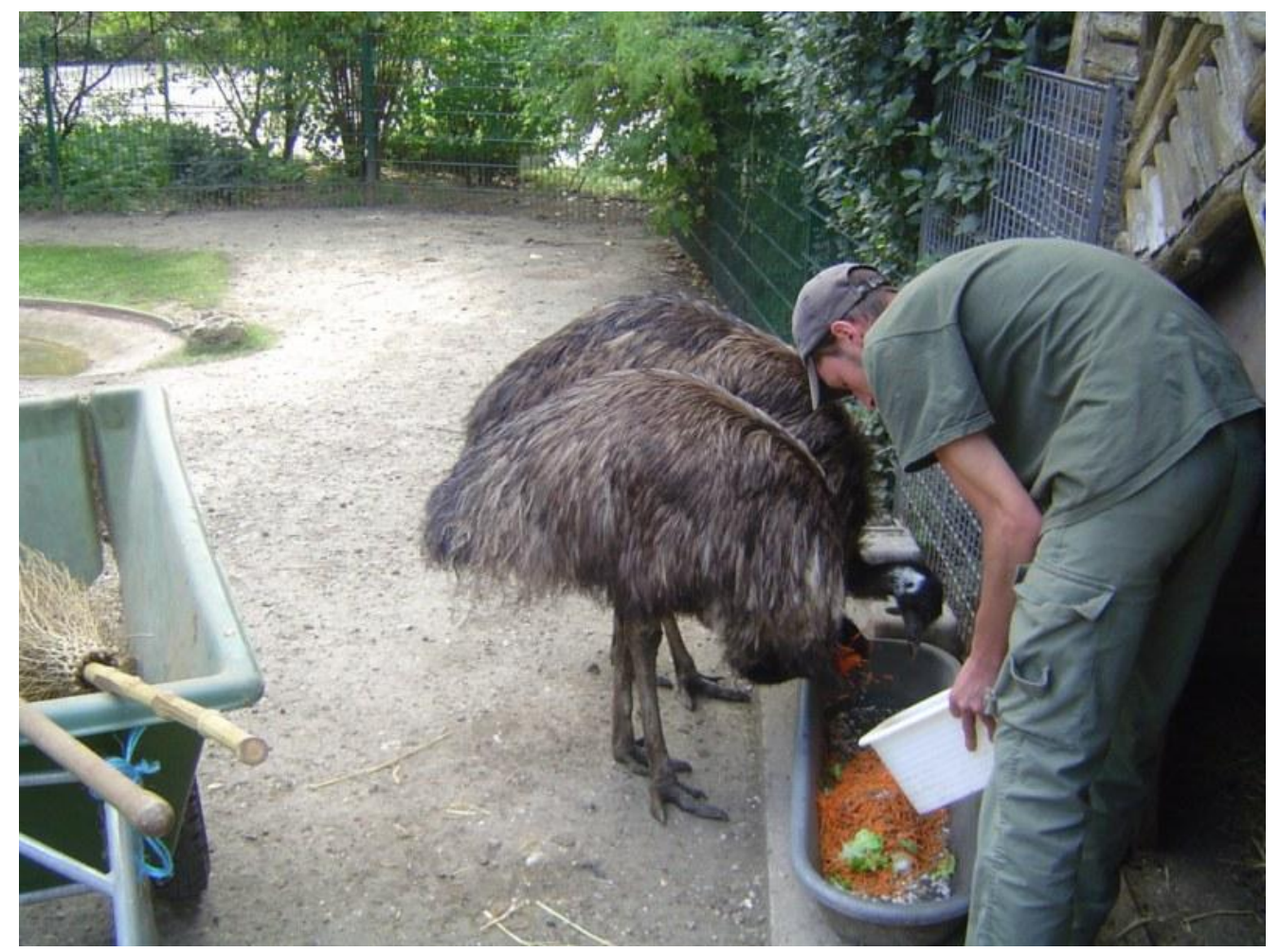

Figure 1 : Lionel déposant la nourriture des émeus, Ménagerie du Jardin des Plantes, 5 septembre 2007

On voit comment les émeus transforment les lieux et les tâches. Un enclos très simple, en terre, avec un petit bassin en béton et quelques zones où l'herbe a résisté aux oiseaux devient

\footnotetext{
${ }^{2}$ Les soigneurs dans le secteur des mammifères marins, par exemple où le nettoyage est souvent beaucoup plus rapide.

${ }^{3}$ Pour une discussion de ces termes voir Estebanez, 2008 ; Marvin, 2008 ; Pellegrini, 1995.

${ }^{4}$ Lionel, environ 25 ans, soigneur, secteur fauverie, Ménagerie du Jardin des Plantes, Paris, 5 septembre 2007.
} 
un semblant de savane australienne. Le balayage, disparaît parce qu'il est fait en compagnie d'animaux. Ce qui est fondamental, c'est bien l'accès privilégié dont les soigneurs disposent : entrer dans l'enclos des animaux est ce qui transforme le travail, ce qui le distingue de l'activité d'un technicien de surface ou d'une ménagère. Cet accès est négocié et susceptible d'être déplacé voire modifié. Ainsi, pour des questions de sécurités, la Ménagerie laisse de moins en moins les soigneurs entrer dans les enclos avec les animaux.

Entrer dans les enclos c'est accéder à un espace réservé dont les limites organisent normalement la séparation entre les humains et les animaux : «Le plus agréable avec le métier de soigneur, c'est le contact avec l'animal. Tu peux pas avec tous, mais bon. Les herbivores, ils aiment bien les caresses. Les reptiles, c'est différent. ${ }^{5}$

Si le contact avec les animaux n'est pas toujours autorisé, il est un attribut de choix : toucher les animaux, c'est établir avec eux une relation particulière.

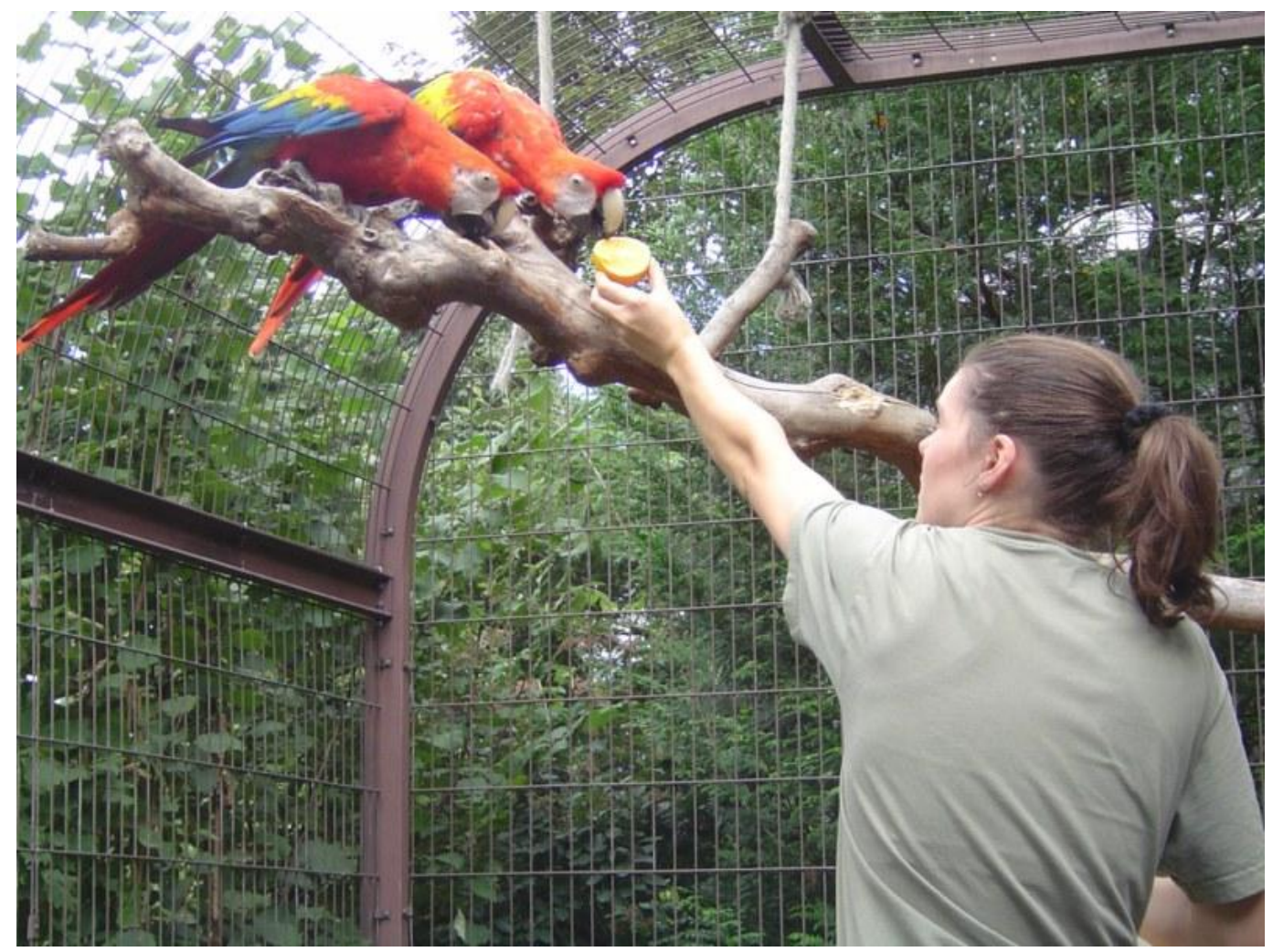

Figure 2 : Betty et deux aras, Ménagerie du Jardin des Plantes, 7 septembre 2007

Ainsi, Betty (figure 2) aurait très bien pu déposer la coupelle de fruits par terre ou dans les branches mais elle choisit de passer quelques instant à donner une tranche d'oranges aux aras. Il y a dans son attention, son regard fixé sur l'ara qui se saisit de l'orange et la tension de son bras mais aussi du corps des perroquets, une marque de la négociation sur la bonne position

\footnotetext{
${ }^{5}$ Ludwig, soigneur, 24/25 ans, secteur du haut, zoo de Pont Scorff, 28 juillet 2007.
} 
de chacun mais également d'un moment privilégié : Betty, tout comme les aras, sont concentrés sur l'action qui se déroule et s'observent à cette occasion.

«Une relation de confiance ?... C'est de l'anthropomorphisme ? Il nous prend pour ses parents. Ils sont là, les hérons, à me faire des poupouilles... » ${ }^{6}$ Certains soigneurs hésitent ainsi, quant au sens à donner à cette relation, poussés en cela par des siècles de pensée de la séparation entre l'humain et l'animal : on voit comment le discours de Sébastien oscille entre une distanciation analytique («l'anthropomorphisme») et l'affectivité de ses relations au quotidien avec les oiseaux qu'il nourrit et qu'il entraine ${ }^{7}$ (« les poupouilles »).

Etre proche des animaux sur de longues périodes, c'est acquérir une connaissance de leur individualité et créer un rapport de confiance qu'ils déclarent être les seuls à avoir : « $\mathrm{Au}$ final, je préfère les rapports que j'ai avec les animaux plutôt que les vétos. C'est plus positif, on les connaît bien, y'a une vraie interaction. Parce que la véto quand elle vient, les animaux ils l'aiment pas, ils crient... Elle vient souvent pour les flécher [...] Elle a besoin de nous parce qu'on connaît les animaux $»^{8}$

En effet, les deux corps de métiers principaux qui ont accès à l'intérieur des enclos et à la proximité avec les animaux sont les soigneurs et les vétérinaires. Une part importante (de l'ordre d'un tiers) des soigneurs auxquels j'ai parlé ont essayé de devenir vétérinaire avant d'être rebutés ou d'échouer face à des études qui ne leur convenaient pas. C'est le cas de Clémence qui compare ici les deux métiers. On voit bien la forme de concurrence qu'elle établit entre les deux champs : elle souligne la relation vraie que les soigneurs établissent avec les animaux par la confiance et la forte interconnaissance qu'ils construisent. Par opposition, le vétérinaire est décrit comme celui qui vient ponctuellement pour effectuer une opération technique, qui plus est, désagréable pour les animaux. Flécher, c'est endormir par une fléchette hypodermique lancée par un fusil spécialement conçu à cet usage. Avec les animaux non domestiques, de nombreuses opérations de contrôle ou de traitement nécessitent une anesthésie du fait du danger potentiel ou plus simplement de l'impossibilité de les manipuler. On observe ainsi en général une grande agitation chez les animaux quand le vétérinaire passe dans les allées.

\section{Une position enviée par le public : un métier fantasmé}

\footnotetext{
${ }^{6}$ Sébastien, soigneur, oiseaux marins, 35 ans, zoo de Pont-Scorff, 28 juillet 2007.

${ }^{7}$ La plupart des oiseaux marins du zoo de Pont-Scorff sont dressés pour un spectacle qui a lieu en été.

${ }^{8}$ Clémence, soigneuse, 22 ans, zoo de Pont Scorff, 1 aout 2007.
} 
Le public d'un zoo vient pour passer du temps avec des animaux vivants, sauvages et exotiques. Au zoo on regarde, on prend des photographies, on imite le cri ou la posture des animaux. On tape sur les barreaux ou sur les vitres et parfois même, on lance des objets ou de la nourriture pour obtenir leur attention. Si les zoos séparent bien entendu les visiteurs des animaux, ils sont conçus pour offrir de la proximité.

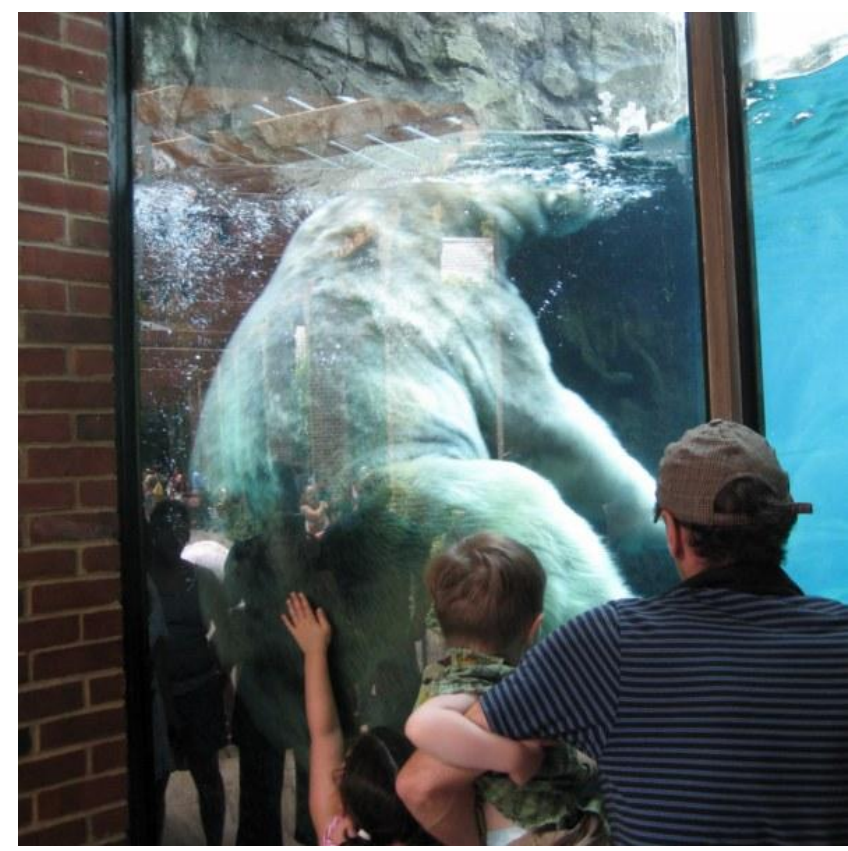

Figure 3 : A la recherche de proximité et d'attention, zoo de Central Park, New York, 10 août 2008

Ce jeu sur la distance, déterminée par la relation aux animaux qui existe au moment de la construction des enclos et des technologies disponibles (Estebanez et Staszak, à paraitre 2010) apparaît également à travers une série de dispositifs de substitution au contact comme des statues en taille réelle à l'extérieur des enclos, des bois ou des morceaux de fourrure.

Comment s'étonner donc de l'aura qui entoure les soigneurs auprès du public ? Les soigneurs sont ceux qui accèdent à l'espace qui est interdit au public. D'une certaine façon, les soigneurs sont perçus comme des sortes de super-visiteurs qui n'ont besoin d'aucun substitut à la proximité, puisqu'ils peuvent accéder aux animaux. Ils dépassent ainsi la contradiction qui traverse les zoos en mettant à distance l'objet même de la visite.

Si l'attention des visiteurs est généralement dirigée vers les animaux, il suffit qu'un soigneur entre dans un enclos ou même passe dans des allées avec de la nourriture ou une brouette chargée d'ustensiles pour qu'ils deviennent le nouveau centre de l'attention. Ainsi, autant les activités pédagogies proposées par exemple à Zoodyssée ou à la Ménagerie du Jardin des 
Plantes font fuir le public, autant les séances menées par des soigneurs à la même Ménagerie attirent les visiteurs : les soigneurs sont ceux qui connaissent réellement les animaux.

«Le soigneur, de l'extérieur est très valorisé. C'est faire des soins, donner des médicaments, caresser les animaux...ils imaginent pas nos horaires et les quantités de fumier qu'on déplace ${ }^{9}$ Les soigneurs combineraient ainsi les attributs du docteur en mission humanitaire et de l'enfant gâté auprès d'un public qui n'aurait aucune conscience de la charge de travail pénible qu'entraîne ce métier. «La vision du soigneur pour les gens, c'est donner à manger [...]. Avant [en passant avec des excréments], c'est: 'oh, c'est dégueulasse'. Et une autre image: 'Oh, vous donnez à manger, c'est bien'. On a l'impression que c'est deux personnages ${ }^{10}$ Comme le souligne Stéphanie, les visiteurs trient les tâches et les images qu'ils ont des soigneurs, sans les rattacher à un même métier, qui se présente alors uniquement sous des traits simplifiés, partiels et souvent imaginaires. La proximité est partagée comme élément de distinction par les soigneurs qui s'en réclament et par le public qui le leur attribue, sans qu'elle renvoie pour autant toujours aux mêmes réalités.

\section{Les dangers de la proximité}

Le public est souvent peu conscient du danger potentiel des animaux (Baratay et HadouinFugier, 1998) ou peut en minimiser l'impact, se sachant en sécurité derrière les systèmes de séparation crées par les zoos. A de rares exceptions près, les animaux ne s'échappent pas de leurs enclos et le public peut ainsi jouer à avoir peur plus que d'avoir réellement peur.

Pour les soigneurs, la proximité est chargée d'autres valeurs. Si elle donne sens au métier, ce n'est pas uniquement par les relations privilégiées qu'elle permet d'établir avec les animaux mais également comme élément de risque important : «Les gens sont inconscients du danger. Sur la fosse aux ours "oh, le gros doudou” [...] Pascal, il a été agressé par une panthère, depuis, il a trop peur [...] il y a toujours une relation de méfiance avec les animaux. C'est un vrai métier dangereux. Dans les parcs, c'est les arrières le problème, la beauté c'est pas le principal $»^{11}$

Être soigneur est un métier dangereux dans lequel des accidents mortels se produisent tous les ans, y compris dans des institutions bien financées ${ }^{12}$. Par ailleurs, la plupart des soigneurs sont

\footnotetext{
${ }^{9}$ Ludwig, soigneur, 24/25 ans, zoo de Pont Scorff, 28 juillet 2007.

${ }^{10}$ Stéphanie, soigneuse, 22/24 ans, zoo de Pont Scorff, 27 juillet 2007.

${ }^{11}$ Stéphanie, soigneuse, 22/24 ans, zoo de Pont Scorff, 27 juillet 2007.

${ }^{12}$ Par exemple, en février 2010, un orque tue une soigneuse au Seaworld d'Orlando, en Floride.
} 
blessés pendant leur carrière, souvent peu gravement, mais parfois avec des conséquences beaucoup plus lourdes. La proximité quotidienne avec les animaux implique une grande attention, en particulier pour éviter la routine, malgré des tâches souvent répétitives, qui peuvent devenir très dangereuses, le cas échéant (mal refermer une grille, s'appuyer sur un loquet...). Les parties de services permettent un accès direct aux animaux avec des systèmes de grilles qui s'ouvrent par de simples systèmes de treuils, manipulables par une personne. Stéphanie, quand nous entrons de nuit dans la loge où se trouve la panthère (cf. figure 4), me précise tout de suite de ne surtout toucher à rien, le treuil commandant la porte d'entrée étant à portée de main, et de me plaquer contre le mur du fond pour éviter de me faire saisir par une patte de l'animal qu'il peut facilement passer sous la grille. Dans la monotonie d'une journée, le passage du soigneur est toujours un moment privilégié pour les animaux qui approchent immédiatement. La panthère est ici en train de jouer avec le balai que passe Stéphanie et par la même occasion teste la grille par de puissants coups de patte.

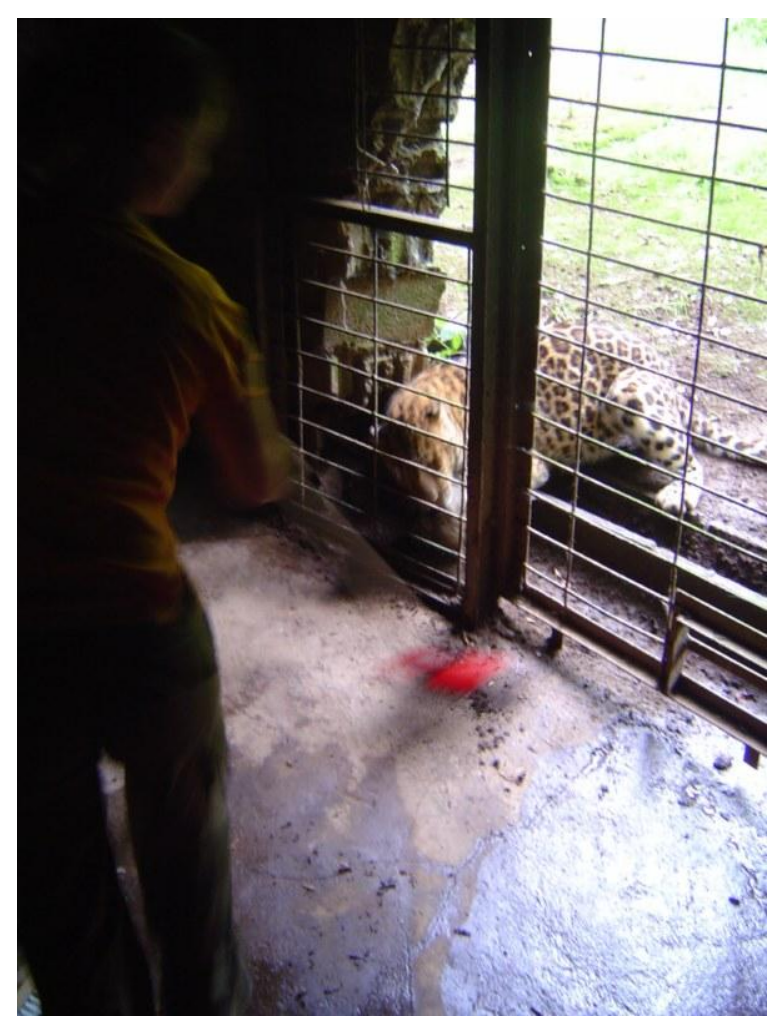

Figure 4 : Stéphanie dans son travail de soigneuse, parfois dangereux, zoo de Pont Scorff, 27 juillet 2007 
Avec les félins, le risque est évident, mais il existe avec l'ensemble des animaux : «Des fois, les oiseaux imprégnés, ils attaquent au visage [...] tant que c'est nous, c'est notre boulot, on accepte. Faut pas qu'il attaque le visiteur. ${ }^{13}$

«L'imprégnation, l'animal à moins peur de l'homme. C'est plus dangereux mais il se cache moins. » ${ }^{14}$ Les animaux aux zoos sont dits «imprégnés » du fait de la présence quasiment continue de l'être humain : d'une certaine façon, c'est la relation à la juste distance qui est modifiée par les allers et venues des visiteurs et les relations privilégiées qui se nouent entre les soigneurs et les animaux. Si les oiseaux que manipule Franck n'étaient pas habitués à sa présence, ils ne se laisseraient pas dresser et refuseraient de participer au spectacle quotidien du zoo. Par ailleurs, des animaux non imprégnés seraient trop stressés par la présence du public et iraient se cacher.

La réduction de la juste distance aux limites du contact - à la fois parce que les animaux sont imprégnés et parce que parfois, les soigneurs vont au-delà de la limite qu'ils auraient du respecter, par exemple, en faisant un geste interprété comme une menace (s'approcher trop d'un petit, avancer trop le bras...) - constitue ainsi à la fois un risque inhérent au métier de soigneur et ce qui en constitue le sens, en les distinguant des autres corps de métier.

\section{Distance et catégories}

\section{Les soigneurs sont-ils trop proches des animaux?}

Si le métier de soigneur est largement fantasmé par le public du fait de la proximité qu'il offre avec les animaux il est également moqué précisément du fait de cette proximité : «T’en as marre du public. Quand t'es dans enclos t'as des réflexions: “Oh l'animal, on lui lance des pop-corns ?" C'est insultant. Quand t'es en cage t'as toujours des réflexions. » ${ }^{15}$; «[Qu'est-ce qui est négatif ?] Le côté chiant du public quoi. Quand t'es dans un enclos, on te jette du popcorn. ${ }^{16}$ La cage fonctionne ici comme un élément de contextualisation : elle transforme les soigneurs en animaux. Ce qui sous-tend les remarques du public, c'est la dichotomie très puissante qui sépare humains et animaux dans la culture dans laquelle ils baignent. Le zoo est un dispositif qui gère un certain niveau de proximité entre humain et animaux sans jamais

\footnotetext{
${ }^{13}$ Franck, soigneur oiseaux marins, 28 ans, zoo de Pont Scorff, 28 juillet 2007.

${ }^{14}$ Aurélie, soigneuse animalière, 25 ans, Zoodysée, 17 juillet 2007.

${ }^{15}$ Stéphanie, soigneuse, 22/24 ans, zoo de Pont Scorff, 27 juillet 2007.

${ }^{16}$ Ludwig, soigneur, 24/25 ans, zoo de Pont Scorff, 28 juillet 2007.
} 
remettre fondamentalement en cause le fait que l'un et l'autre appartiennent à des mondes différents. Un soigneur dans une cage est ainsi à la fois source d'intérêt (Que fait-il ? Comment réagissent les animaux à leur arrivée ?) et une bonne occasion de s'amuser à peu de frais : le fait qu'on leur lance du pop-corn est clairement perçu par les soigneurs comme une façon de les humilier en les rejetant hors de l'humanité.

On voit ainsi comment la distance et le franchissement de certains seuils, dans ce contexte à un impact direct sur la façon dont on décrit et on traite les soigneurs. Cet impact concerne également les animaux et la relation qui existe entre eux et les soigneurs.

\section{Le proche est-il encore le sauvage?}

«Le lion ne se fait caresser que s'il veut. L'animal de zoo, il est sauvage avec une relation privilégiée aux humains. Il est près du retour à l'état sauvage ${ }^{17} ;$ «Avec les fauves, ça se joue beaucoup sur un rapport de domination [...] Ils sont dangereux s'ils sont trop imprégnés. La peur de l'animal sauvage pour l'homme disparaît. ${ }^{18}$ Dans le même zoo, deux visions diamétralement opposées des mêmes animaux transparaît à travers ces citations. D’un côté, les lions sont toujours prêts à retourner à l'indépendance totale et la proximité que les soigneurs ou les dresseurs entretiennent avec eux n'est que provisoire. Les lions sont dangereux, car ils sont incontrôlables, toujours prêts à reprendre leur véritable nature, loin des humains. Le retour à l'état sauvage renvoie à l'incapacité des humains de les transformer durablement. Au contraire, la seconde position montre combien l'impact des humains est important dans la transformation des lions. Des lions imprégnés deviennent dangereux parce qu'ils sont trop proches des humains. Les soigneurs auraient ainsi un impact définitif sur les animaux dont ils s'occupent et dont ils deviennent les pairs ou tout du moins les êtres avec lesquels ils ont le plus de relations.

«On se fait passer pour la mère de l'animal. Si je le remets, il se fait bouffer [par sa mère]. Il ignore l'autre animal, il vient nous voir. [...] ça devient délicat le rapport homme animal. » ${ }^{19}$ Au-delà du lien d'espèce, Christèle souligne l'importance du lien social qui se développe entre les soigneurs et leurs animaux. Leur statut est sans doute unique : nés et échangés dans le système des zoos, ils ont souvent comme plus proche relation, en dehors des animaux avec lesquels ils partagent leur enclos, leurs soigneurs. Comme le souligne G. Marvin (2008), ils ne

\footnotetext{
${ }^{17}$ A. Le Héritte, directeur du zoo de Pont Scorff, ancien dresseur, Jeudi 26 juillet 2007.

${ }^{18}$ Anthony, soigneur, responsable des spectacles, 35 ans, Pont Scorff, 29 juillet 2007.

${ }^{19}$ Christèle, soigneuse, mammifères marins, zoo de Pont Scorff, 24/25 ans, 29 juillet 2007.
} 
sont ni sauvages, ni domestiques mais relèvent d'un autre régime de classification et d'identité lié au dispositif dans lequel ils évoluent

Pour autant, la plupart des zoos ont adhéré à un discours officiel qui met en avant le rôle de protection et de conservation d'espèce en voie de disparition ${ }^{20}$. Leur existence se justifierait ainsi par leur capacité à maintenir un nombre suffisant de spécimens de certaines espèces pour pouvoir les relâcher une fois leur environnement protégé. Ce discours est largement illusoire, mais il implique pour certain d'entre eux, de limiter autant que possible les transformations que la captivité et la proximité avec les humains induit. La question du contact est ainsi un enjeu qui touche à la fonction même des zoos. Si certains d'entre eux (par exemple le Howletts Wild Animal Park, en Angleterre) encouragent leurs soigneurs à entrer dans les enclos de tous les animaux et à les toucher, la règle est bien plus souvent de l'éviter au maximum.

«On a pas le droit de toucher les animaux [...]. Il faut garder une distance avec les animaux pour les garder le plus sauvage, le plus authentique [possible]. On est pas là pour caresser. $»^{21}$ Ce qui frappe dans le discours d'Héloïse est le lien qu'elle fait entre la distance matérielle et les catégories. Etre trop près et, pire encore, toucher modifie l'être des animaux qui perdent ainsi leur authenticité d'animaux sauvages, soulignant la puissance attribuée au contact..

\section{Conclusion}

La position et la définition des acteurs s'établie de manière relationnelle, autour du dispositif du zoo qui permet et organise la proximité par une série de systèmes techniques de fosses, de barreaux mais aussi de règlements (les soigneurs peuvent ou non toucher les animaux; le public est ou non autorisé à les nourrir).

Les soigneurs, souvent dévalorisés dans la hiérarchie des zoos, dont ils occupent la plus basse place, éclairent pourtant l'enjeu principal qui les traverse : la négociation d'une juste distance entre des animaux sauvages et exotiques et des humains. Ils possèdent à la fois une position enviée par le public, qui doit se contenter d'un contact par substitution, et d'une situation étrange dans laquelle leur humanité est questionnée par les lieux où ils se trouvent et leur action remise en cause comme facteur de dénaturation des animauxes animaux. Ce regard sur les soigneurs permet enfin de souligner l'importance de l'attention au niveau micro, où se

\footnotetext{
${ }^{20}$ Voir à ce sujet le site de la World Association of Zoos and Aquariums (http://www.waza.org/en/site/conservation, accédé le 13 mars 2010) ou le livre d'un influent directeur de zoo (Hancocks, 2001)

${ }^{21}$ Héloïse, 22 ans, soigneuse, Ménagerie du Jardin des Plantes, 4 septembre 2007.
} 
joue le sens de leurs échanges : distance matérielle et symbolique y apparaissent comme les deux faces d'un même tout.

\section{Bibliographie}

K. Anderson, "Culture and nature at the Adelaïde zoo: at the frontier of 'human' geography », Transaction of the Institute of British Geographers, 20, pp. 275-294, 1995.

É. Baratay et É. Hadouin-Fugier É, Zoos. Histoire des jardins zoologiques en Occident (XVIeXXe siècles), La Découverte, Paris, 1998.

J. Berger J., About Looking, Pantheon Books, New York, 1980.

J. Estebanez et J.-F. Staszak J.-F., «Animaux humains et non-humains au zoo. L'expérience de la frontière animale », in Dubied A., Fall J. et Gerber D. (dir.), La Frontière Humain/animal, Droz, Genève, 2010 (à paraître).

J. Estebanez, «Les jardins zoologiques ou l'exotique à portée de main », Le Globe, 148, pp. 89-105, 2008.

J. Estebanez, «Les jardins zoologiques et la ville : quelle nature pour le Biodôme de Montréal? », Annales de géographie, 652, pp. 708-731, 2006.

D. Hancocks, A different nature : the paradoxical world of zoos and their uncertain future, University of California Press, Berkeley, 2001.

G. Marvin, «L'animal de Zoo. Un rôle entre sauvage et domestique », Techniques et culture, 50, pp. 103-119, 2008.

B. Mullan et G. Marvin, Zoo Culture, University of Illinois Press, Urbana and Chicago, 1999.

N. Rothfels, Savages and Beasts: the Birth of the Modern Zoo, Johns Hopkins University Press, Baltimore, 2002.

P. Pellegrini, «Zoos, parcs et réserves » in B. Lizet (dir.), Des bêtes et des hommes. Le rapport à l'animal : un jeu sur la distance, CTHS, Paris, 1995. 